\title{
Bilateral Antrochoanal Polyp in an Adult: A Rarity
}

\author{
${ }^{1}$ Pawan Singhal, ${ }^{2}$ Nitin Gupta \\ ${ }^{1}$ Assistant Professor, Department of ENT and Head and Neck Surgery, SMS Medical College, Jaipur, Rajasthan, India \\ ${ }^{2}$ Ex-Resident, Department of ENT, Government Medical College, Kota, Rajasthan, India
}

Correspondence: Pawan Singhal, Assistant Professor, Department of ENT and Head and Neck Surgery, SMS Medical College Jaipur, Rajasthan, India, e-mail: drps.ent@gmail.com

\section{ABSTRACT}

Antrochoanal polyp is a benign solitary polypoidal lesion which arises in maxillary sinus. It appears radiologically as an opacifying and enlarging lesion in the sinus cavity without bony destruction. They are almost always unilateral and occur more commonly in children and young adults. Here we report a case of bilateral antrochoanal polyp (ACP) in an adult because of its rarity.

Keywords: Antrochoanal polyp, CT scan, Bilateral AC polyp.

\section{INTRODUCTION}

Antrochoanal polyps usually present unilaterally. It is defined as a polyp originating in the maxillary sinus, protruding in the middle meatus through the ethmoidal infundibulum or an accessory ostium and further extends posteriorly through the choana into the nasopharynx or in oropharynx in a few cases. An antrochoanal polyp usually has three parts according to its extension, i.e antral, nasal and choanal part. It is seen commonly in children and in both males and females. Exact etiology is not known but probably chronic infection of the maxillary sinus may be one of the factors in pathogenesis of AC polyps. Antrochoanal polyp presenting in both nasal cavities simultaneously is very rare occurrence.

\section{CASE REPORT}

A 32-year-old female presented to us with the chief complaint of bilateral progressive nasal obstruction for the past 2 years. She was also complaining of headache and heaviness in the head for the last 6 months. There was increase in the intensity of symptoms for the last 2 months and causing difficulty in breathing while sleeping.

General physical examination of that patient was within normal limits. There was no personal or family history of allergy, asthma, diabetes and tuberculosis and the patient was normotensive.

Anterior rhinoscopy revealed pale masses in both nasal cavities. The size was not so apparent as it was lying more posteriorly in the nasal cavity. The mass in the left nasal cavity was larger than the right side. Posterior rhinoscopy revealed pale polypoidal masses in both choana. The left side mass was larger and hanging in the nasopharynx.

Skiagram of PNS water's view showed haziness/opacity in the both maxillary antrum and nasal cavities. CT scan of nose and paranasal sinuses (Fig. 1) showed soft tissue mass in both maxillary sinuses. The osteomeatal opening of both maxillary sinuses were widened and blocked. The soft tissue masses were extending into both nasal cavities and nasopharynx and appeared more prominent on left side with fluid density in ethmoidal air cells. Routine blood, urine and radiological investigations were within normal limits.

The polyps were removed under general anesthesia with the help of nasal endoscopes. The polyps were pale, white in color and had smooth glossy surface (Fig. 2). They were soft in consistency. The gross appearance showed that both polyps had three parts—antral, nasal and choanal.

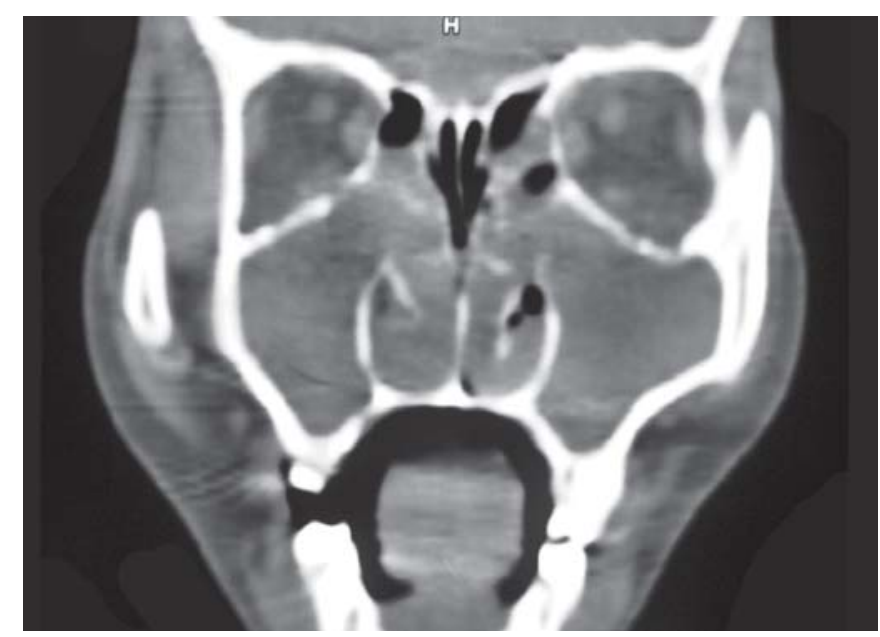

Fig. 1: CT scan PNS showing bilateral antrochoanal polyp 


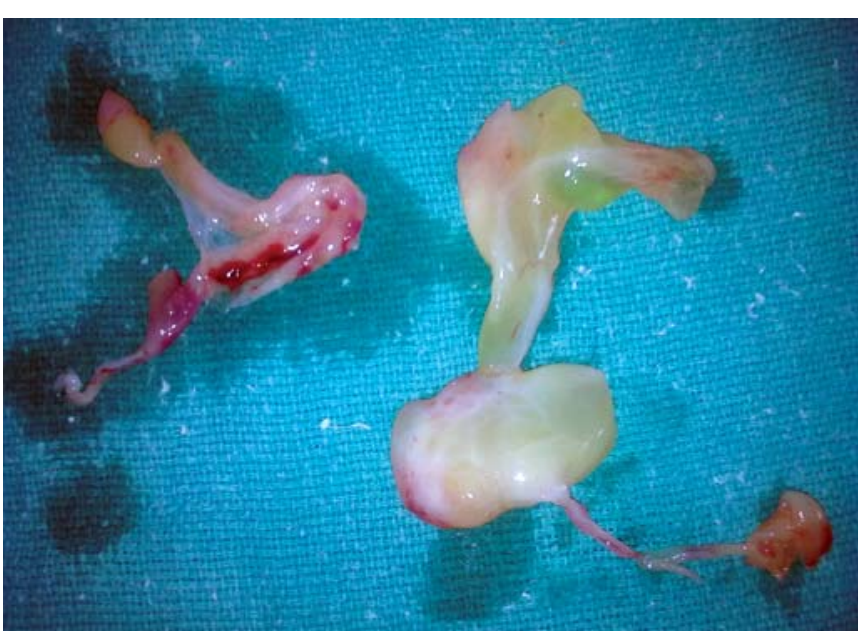

Fig. 2: Polyp removed from both nasal cavity

Histopathology showed it to be benign inflammatory nasal polyps. Final diagnosis was bilateral antrochoanal polyp.

\section{DISCUSSION}

Antrochoanal polyp (ACP) is said to be unilateral and seen mostly in children. They manifest as unilateral nasal blockade, headache or heaviness in head, nasal discharge, postnasal drip, anosmia or hyposmia. Symptoms of allergy are not commonly seen in these patients but may present sometimes.

Bilateral antrochoanal polyps are extremely rare. ${ }^{1-4}$ So far, only four cases have been reported in the literature. The presented case was a 32-year-old female who was otherwise healthy with no history of allergy and asthma. The diagnosis of bilateral antrochoanal polyps was made on the basis of careful clinical examination and radiological modalities. Skiagram of PNS gives some idea about diagnosis but CECT is a better modality in making diagnosis $^{5}$ and should always be supplemented with diagnostic nasal endoscopy. ${ }^{6}$ Antrochoanal polyp usually appears as nonenhancing mass on CT scan unless they are infected. They occupy the osteomeatal complex and cause widening of maxillary ostea. ${ }^{6,7}$

Nasal endoscopy is gold standard tool in making diagnosis and nowadays functional endoscopic sinus surgery (FESS) is the treatment of choice for AC polyp. Cook et al reported that FESS is the recommended surgical treatment method and showed no recurrence with minimal complications in a series of 33 adult patients. ${ }^{8}$

The previously done Caldwel-Luc operation for AC polyps is not preferred nowadays and is only indicated in recurrent cases. Regular follow-up of the patient after surgery is necessary to see for any recurrence.

\section{CONCLUSION}

Antrochoanal polyps are generally unilateral in occurrence and seen mostly in children and adolescents. Bilateral occurrence of antrochoanal polyp in an adult is a very rare incidence.

\section{REFERENCES}

1. Myatt HM, Cabrera M. Bilateral antro-choanal polyp in a child: A case report. J Laryngol Otol 1996;110:272-74.

2. Basu SK, Bandyopadhyay SN, Bora H. Bilateral antro-choanal polyps. J Laryngol Otol 2001;115(7):561-62.

3. Sami Jmeian. Bilateral antrochoanal polyps in a child: An extremely rare case. JRMS 2006;13(2):57-58.

4. Yilmaz YF, Titiz A, Ozean M, Tezer MS, Ozlugedik S, Unal A. Bilateral antrochoanal polyp in an Adult: A case report. B-ENT ISSN 1781-1782, 2007;3(2):97-99.

5. Towbin R, Dunbar JS, Bove K. Antrochoanal polyp. Am J Roentgenol 1979;132(1):27-31.

6. Chen JM, Schloss MD, Azouz NE. Antro-choanal polyp: A 10year retrospective study in the paediatric population with a review of the literature. J Otolaryngol 1989;18(40):168-72.

7. Kamel R. Endoscopic transnasal surgery in antrochoanal polyp. Arch Otolaryngol Head Neck Surg 1990;116(7):841-43.

8. Cook PR, Davis WE, McDonald R, McKinsey JP. Antrochoanal polyposis: A review of 33 cases. ENT J 1993;72(6):401-02, 404-10. 